\title{
Small-emittance and low-beta lattice designs and optimizations
}

\author{
C. Sun, * D. S. Robin, H. Nishimura, C. Steier, and W. Wan \\ Lawrence Berkeley National Laboratory, Advanced Light Source, Berkeley, California 94720-8228, USA
}

(Received 31 January 2012; published 4 May 2012)

This paper uses the advanced light source (ALS) storage-ring lattice as an example to illustrate the strategies and techniques that we developed for lattice design and optimization. First, the theoretical minimum emittance (TME) theory is applied to optimize the ALS storage-ring lattice for its future upgrades. The study confirms the results found in earlier study using both global scan of all stable settings and multiobjective genetic algorithms (MOGA) techniques. It is shown that, using TME, the ALS natural emittance can be reduced to an even smaller value by introducing additional quadrupoles to the straight, which is unknown in previous studies. Then, the nonlinear properties of the lattice are optimized using MOGA. Instead of the conventionally used dynamic aperture area, the total diffusion rate of the lattice is used as an objective in the optimization, which leads to a superior performance in nonlinear beam dynamics. Finally, to find a best overall working lattice for ALS future upgrades, the linear and nonlinear properties of the lattice are optimized simultaneously using MOGA. Compared to the widely used dynamic aperture tune scan technique, MOGA not only allows us to rapidly find a best working point in a wide searching range, but also provides us trade-offs among the optimization objectives, such as the low emittance, small beta function, and large dynamic aperture. These trade-offs give us a guideline to choose a candidate lattice for ALS future upgrades. The strategies and techniques presented in this paper are not limited to the ALS, and can be adopted to other facilities.

DOI: 10.1103/PhysRevSTAB.15.054001

PACS numbers: 29.20.db, 41.85.- p

\section{INTRODUCTION}

The advanced light source (ALS) at Lawrence Berkeley National Laboratory is one of the earliest 3rd generation light sources. Since the commissioning in 1993 [1], a series of upgrades have been successfully completed, including the installation of superconducting bend magnets (Superbends) in 2001 [2] and implementation of top-off injection in 2007 [3]. To keep the ALS competitive in the future, it was recognized a few years ago that further upgrades to lower the storage-ring emittance will be necessary $[4,5]$.

The ALS low-emittance upgrade project began in 2009. When this upgrade is finished, the horizontal emittance will be reduced by three factors from current $6.3 \mathrm{~nm}$ rad to about $2 \mathrm{~nm}$ rad. Figure 1 shows optics functions of one ALS sector before and after the upgrade. The entire ALS storage ring consists of 12 sectors, and each sector is a triple bend achromat with a mirror symmetric structure consisting of 3 families of quadrupoles ("QF," "QD," and "QFA"). Figure 1(a) shows the optics functions of the current lattice; 1(b) shows the optics functions of the upgraded lattice. From the plots, we can see the major quantitative changes of the optics functions are the horizontal beta and dispersion functions which are in-

\section{*CCSun@lbl.gov}

Published by the American Physical Society under the terms of the Creative Commons Attribution 3.0 License. Further distribution of this work must maintain attribution to the author(s) and the published article's title, journal citation, and DOI. creased in the straights. This upgrade project (so-called baseline upgrade) is under way and will improve the brightness of many beam lines by several factors after the completion [6].

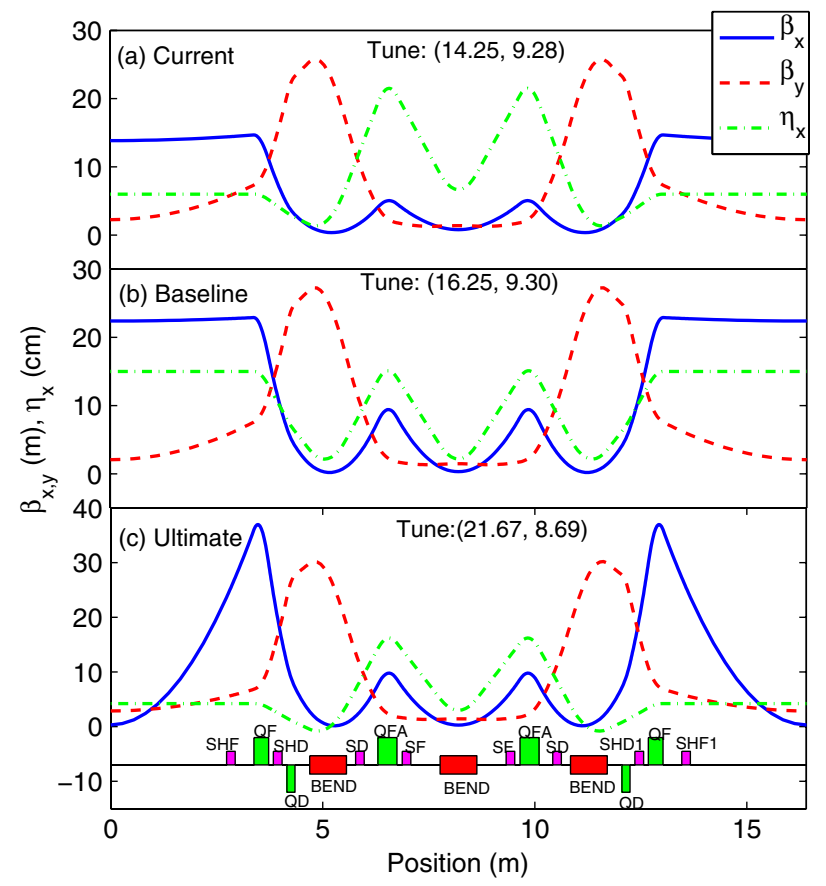

FIG. 1. Layout of one sector of ALS lattice and its associated optics functions for (a) current lattice, (b) baseline upgrade, (c) ultimate upgrade. 
It was quickly realized that the baseline upgrade lattice does not provide "ultimate" insertion device brightness due to the phase space mismatch of the electron and photon beams and also the large dispersion functions in the center of straights. If we could reduce the horizontal beta and dispersion functions to small values at the center of straight, i.e., upgrade the lattice to the one shown in Fig. 1 (c) (ultimate upgrade), the insertion device brightness could be improved by another 2 or 3 factors.

This paper presents strategies and techniques that we developed to optimize the linear and nonlinear properties of the ALS storage-ring lattice for its future potential upgrades. In Sec. II, we first discuss the optimal beta function at the center of straight to maximize insertion device brightness. In Sec. III, we apply the theoretical minimum emittance (TME) theory [7,8] to search for small-emittance and low-beta lattices. The study confirms the results found in earlier study using both global scan of all stable settings (GLASS) [9] and multiobjective genetic algorithms (MOGA) [10] techniques. We show that, using TME, we can reduce the ALS emittance to an even smaller value by introducing additional quads to the straight, which is unknown in previous studies. In Sec. IV, the MOGA is applied to optimize dynamic aperture of ALS upgrade lattices. Instead of the conventionally used dynamic aperture area, we used total diffusion rate as an objective to optimize the lattice. It is illustrated that this optimization can lead to a superior performance in nonlinear beam dynamics. To find a best overall working lattice for ALS future upgrades, in Sec. V, we optimize the linear and nonlinear properties of the lattice simultaneously using MOGA. Compared to the widely used dynamic aperture tune scan (DATS) technique, MOGA not only allows us to rapidly find a best overall solution in a wide searching range, but also provides us trade-offs among the lower emittance, small beta function and larger dynamic aperture. Finally, we conclude the paper in Sec. VI.

\section{OPTIMAL BETA FUNCTION}

High brightness of insertion device (ID) is one of the most desirable properties of the synchrotron radiation light source, and a great deal of effort is devoted to optimizing it. An approximate formula for the brightness of undulator radiation is given by

$$
B=\frac{F}{4 \pi^{2} \Sigma_{x} \Sigma_{x^{\prime}} \Sigma_{y} \Sigma_{y^{\prime}}}=\frac{F}{4 \pi^{2} \varepsilon_{x}^{\mathrm{eff}} \varepsilon_{y}^{\mathrm{eff}}},
$$

where $F$ is the photon flux; $\Sigma_{x, y}$ and $\Sigma_{x^{\prime}, y^{\prime}}$ are the effective rms photon beam size and divergence, which are given by the convolution of the electron beam and diffractionlimited photon beam sizes and divergences; $\varepsilon_{x, y}^{\text {eff }} \equiv$ $\Sigma_{x, y} \Sigma_{x^{\prime}, y^{\prime}}$ are the effective photon beam emittances. Assuming the electron beam and photon beam have Gaussian distributions, $\Sigma_{x, y}$ and $\Sigma_{x^{\prime}, y^{\prime}}$ are given by

$$
\begin{aligned}
\Sigma_{x, y} & =\sqrt{\sigma_{x, y}^{2}+\sigma_{r}^{2}}, \quad \Sigma_{x^{\prime}, y^{\prime}}=\sqrt{\sigma_{x^{\prime}, y^{\prime}}^{2}+\sigma_{r^{\prime}}^{2}} \\
\sigma_{x, y} & =\sqrt{\epsilon_{x, y} \beta_{x, y}+\sigma_{E}^{2} \eta_{x, y}^{2}}, \quad \sigma_{x^{\prime}, y^{\prime}}=\sqrt{\epsilon_{x, y} \gamma_{x, y}+\sigma_{E}^{2} \eta_{x, y}^{\prime 2}}, \\
\sigma_{r} & =\frac{1}{2 \pi} \sqrt{\frac{\lambda L}{2}}, \quad \sigma_{r}^{\prime}=\sqrt{\frac{\lambda}{2 L}}
\end{aligned}
$$

where $\sigma_{x, y}$ are the electron beam size and divergence including the dispersion effect; and $\sigma_{r, r^{\prime}}$ are the diffraction-limited photon beam size and divergence. $\boldsymbol{\epsilon}_{x, y}$ and $\sigma_{E}$ are the electron beam emittance and energy spread, $\beta_{x, y}$ and $\gamma_{x, y}$ are Twiss functions, and $\eta_{x, y}$ and $\eta_{x, y}^{\prime}$ are dispersion functions. $L$ is the undulator length and $\lambda$ is the radiation wavelength.

At the center of the ID straight, usually $\gamma_{x, y}=1 / \beta_{x, y}$, $\eta_{x, y}^{\prime}=0$, and the effective photon beam emittances are given by

$$
\varepsilon_{x, y}^{\mathrm{eff}} \equiv \Sigma_{x, y} \Sigma_{x^{\prime}, y^{\prime}}=\sqrt{\epsilon_{x, y}^{2}+\epsilon_{x, y}\left(\beta_{x, y}+\frac{L^{2} / 4 \pi^{2}+\sigma_{E}^{2} \eta_{x, y}^{2} \frac{2 L}{\lambda}}{\beta_{x, y}}\right) \frac{\lambda}{2 L}+\frac{\lambda^{2}}{16 \pi^{2}}+\frac{\lambda}{2 L} \sigma_{E}^{2} \eta_{x, y}^{2}}
$$

To maximize the undulator brightness, we need to minimize $\varepsilon_{x, y}^{\text {eff }}$, which has been discussed in papers [11-13]. However, in these papers, the contributions of the diffraction-limited photon beam size and divergence $\sigma_{r, r^{\prime}}$ are neglected. This is a good approximation for a third generation hard $\mathrm{x}$-ray light source. However, for a soft xray light source, such as the ALS, where the diffractionlimited photon beam size and divergence are comparable to the electron beam size and divergence, we need to take into account these contributions in $\varepsilon_{x, y}^{\text {eff }}$.

Taking a further step to minimize Eq. (3), it is obvious that first we need to minimize the electron beam emittance $\epsilon_{x, y}$. Then, another question arises: what are the optimal $\beta_{x, y}$ functions at the location of undulator? To answer this question, we need to minimize $\varepsilon_{x, y}^{\text {eff }}$ as a function of $\beta_{x, y}$, which yields the optimal beta value given by

$$
\beta_{x, y}^{\mathrm{opt}}=\sqrt{\frac{L^{2}}{4 \pi^{2}}+\frac{2 L}{\lambda} \sigma_{E}^{2} \eta_{x, y}^{2}}
$$

Here, we assume that the beta function is independent of the emittance and the second order term of $\frac{\lambda}{2 L} \sigma_{E}^{2} \eta_{x, y}^{2}$ in Eq. (3) is negligible. We can see that this optimal beta function depends on the length of the undulator, wavelength of the radiation photon, energy spread, and 
dispersion functions of the electron beam. For dispersion free lattice, $\eta_{x, y}=0$, the optimal beta function is reduced to the well-known value $\beta_{x, y}^{\text {opt }}=L / 2 \pi$.

It should be noted that Eqs. (1)-(4) are based upon the assumption of Gaussian distributions of the electron and photon beams. However, this assumption may be too crude, and the undulator brightness given by Eq. (1) is not accurate. A more rigorous calculation can be carried out using numerical synchrotron radiation codes, such as SDDSBRIGHTNESS/URGENT [14] and SPECTRA [15]. Using these codes, we can scan the undulator radiation brightness for different $\beta_{x}$ values, and determine the optimal beta function more accurately. The results are shown in Fig. 2. Three types of ALS lattice (current, baseline, and ultimate upgrade) are studied. We can see that the optimal beta values $\beta_{x}^{\text {opt }}$ given by the peak location of the brightness curve are different from the values given by Eq. (4).

In conclusion of this section, to achieve a high brightness of insertion device, we need not only minimize the emittance of the beam, but also optimize the beta functions at the center of straights. Especially, when the electron beam emittance is small and its beam size is comparable to the diffraction-limited photon beam size, the optimization of the beta function can significantly improve the brightness of insertion device. Generally speaking, a low-beta value is desirable since the phase spaces of the electron and photon beams have a better match, which results in a small effective photon beam size and divergence. From Fig. 2, we can see that the

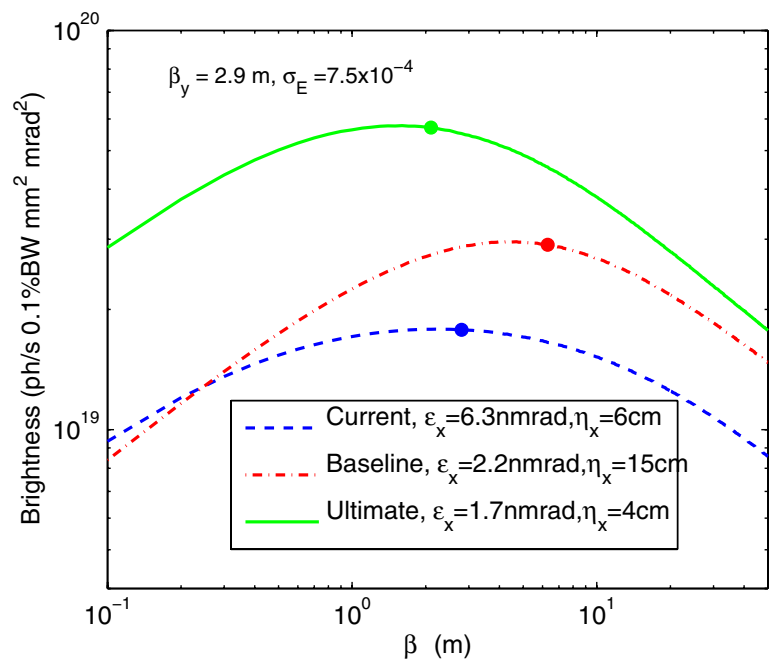

FIG. 2. Undulator brightness as functions of the $\beta_{x}$ for three different type lattices. The numerical synchrotron radiation code SDDSBRIGHTNESS/URGENT is used for the calculation. The brightness is calculated at a photon wavelength of $3 \mathrm{~nm}$ for an undulator with the period length of $50 \mathrm{~mm}$, and total length of $4.45 \mathrm{~m}$. The dots represent the locations of optimal beta calculated using Eq. (4). optimal beta value is about 1 meter for ALS ultimate upgrade lattice. It should be noted that there is no single optimal beta value for all types of ALS lattices because it depends on the dispersion function which is different for different type lattices. However, for current and baseline type lattices, the brightness has a weak dependence on the beta value around the peak region (1-10 meters) as shown in Fig. 2. Therefore, 1 meter is a good choice for all types of lattices. In the following sections, we are going to use the beta function of 1 meter as an objective to optimize ALS lattices.

\section{SMALL-EMITTANCE AND LOW-BETA LATTICE DESIGN}

\section{A. Optimization techniques}

From previous discussion, we know that to achieve high brightness of insertion devices it is desirable to design a storage ring with a small-emittance and low-beta function (about 1 meter). At the ALS, two techniques, global scan all stable setting (GLASS) [9] and MOGA [10], have been successfully developed to search for small-emittance and low-beta lattice for future upgrades. To use the technique of GLASS, we first scan all possible quadrupole settings and find all stable ones; then compute properties of all stable settings and filter by properties those settings that may be of interest. This technique is straightforward and easily implemented for a simple lattice with a small number of quadrupole families (usually, less than 4). However, for a lattice with a large number of variables, this technique is unfeasible because the computing time exponentially depends on the number of the variables. To address this limitation, MOGA were successfully developed at the ALS. MOGA is a method to generate optimal solutions using a technique inspired by natural evolution. The optimization begins by randomly creating a population of possible settings, and then evolves them in subsequent generations by mimicking natural evolution process such as inheritance, mutation, selection, and crossover. In the field of particle accelerator, genetic algorithms have been successfully applied to optimize superconducting magnets [16], injection transport lines [17], photoninjector [18], storage-ring linear lattice [10], and dynamic aperture [19].

To search for an optimal lattice, the starting points of both GLASS and MOGA are quadrupole strengths which are either uniformly or randomly selected, then lattice properties are calculated for each quadrupole setting, and the one with optimal properties is selected. Based upon the TME theory $[7,8]$, we recently developed a different approach to search for small-emittance and low-beta lattices. Instead of the quadrupole strengths, the starting point of this method is the lattice properties; once optimal lattice properties are found, the quadrupole strengths are determined by lattice matching. In this section, we present the details of this method and apply it to optimize ALS storage-ring lattice. 


\section{B. Horizontal natural emittance}

It is well known that the horizontal natural emittance of a storage ring is given by

$$
\epsilon_{x}=C_{q} \gamma^{2} \frac{\left\langle\mathcal{H}(s) /|\rho(s)|^{3}\right\rangle}{J_{x}\left\langle 1 / \rho(s)^{2}\right\rangle},
$$

where $C_{q}=3.832 \times 10^{-13} \mathrm{~m} ; \gamma=E / m c^{2}$ is the Lorentz factor of the electron beam; $J_{x}$ is the horizontal partition factor; $\rho(s)$ is the bending radius; $s$ represents the longitudinal position along the ring; and the brackets " \langle\rangle " mean averaging over the storage ring; the $\mathcal{H}(s)$ function is given by

$$
\mathcal{H}(s)=\gamma_{x} \eta_{x}^{2}+2 \alpha_{x} \eta_{x} \eta_{x}^{\prime}+\beta_{x} \eta_{x}^{\prime 2}
$$

where $\beta_{x}, \alpha_{x}$, and $\gamma_{x}$ are Twiss functions of the beam; and $\eta_{x}$ and $\eta_{x}^{\prime}$ are dispersion functions.

For an isomagnetic storage ring which has identical bending magnets, Eq. (5) can be simplified to

$$
\epsilon_{x}=\frac{C_{q}}{J_{x}} \frac{\gamma^{2}}{\rho} \frac{1}{N} \sum\langle\mathcal{H}(s)\rangle_{\mathrm{dipole}}
$$

where $N$ is the number of dipoles in the ring, $\sum$ means summations of all the dipoles, and $\langle\mathcal{H}(s)\rangle_{\text {dipole }}$ is the average $\mathcal{H}$ function over the dipole. For a sector bend, it is given by [20]

$$
\begin{aligned}
\langle\mathcal{H}(s)\rangle_{\text {dipole }}= & \gamma_{0} \eta_{0}^{2}+2 \alpha_{0} \eta_{0} \eta_{0}^{\prime}+\beta_{0} \eta_{0}^{\prime 2}+\frac{2 l}{\rho}\left\{-\left(\gamma_{0} \eta_{0}+\alpha_{0} \eta_{0}^{\prime}\right) \frac{k l-\sin k l}{k^{3} l^{2}}+\left(\alpha_{0} \eta_{0}+\beta_{0} \eta_{0}^{\prime}\right) \frac{1-\cos k l}{k^{2} l^{2}}\right\} \\
& +\frac{l^{2}}{\rho^{2}}\left\{\gamma_{0} \frac{3 k l-4 \sin k l+\sin k l \cos k l}{2 k^{5} k^{3}}-\alpha_{0} \frac{(1-\cos k l)^{2}}{k^{4} l^{3}}+\beta_{0} \frac{k l-\cos k l \sin k l}{2 k^{3} l^{3}}\right\},
\end{aligned}
$$

where $\beta_{0}, \alpha_{0}, \gamma_{0}, \eta_{0}$, and $\eta_{0}^{\prime}$ are Twiss and dispersion functions of the beam at the entrance of the dipole; $l$ is the length of the dipole; and $k$ is the focusing function of the dipole. For a rectangular bend, the design orbit of electron beam is not normal to the edges of the magnet, and thus it contains an extra focusing/defocusing effect at the edges. We need to replace $\alpha_{0}$ and $\eta_{0}^{\prime}$ in Eq. (8) by [20]

$$
\alpha_{1}=\alpha_{0}-\frac{\beta_{0}}{\rho} \tan \phi, \quad \eta_{1}^{\prime}=\eta_{0}^{\prime}+\frac{\eta_{0}}{\rho} \tan \phi,
$$

where $\phi$ is the angle of the design orbit with respect to the edges of the dipole.

\section{ALS lattice design}

Since the basic ALS sector has a mirror symmetric triple bend structure (Fig. 1), the two outer bends have the same average $\mathcal{H}$ functions. Thus, the horizontal emittance of the ALS storage ring is given by

$$
\epsilon_{x}\left(\beta_{0}, \alpha_{0}, \eta_{0}, \eta_{0}^{\prime}, k_{q f a}\right)=\frac{C_{q} \gamma^{2}}{\rho J_{x}}\left(\frac{2}{3}\langle\mathcal{H}\rangle_{o}+\frac{1}{3}\langle\mathcal{H}\rangle_{i}\right),
$$

where the subscripts $o$ and $i$ are used to identify the outer and inner bends. Given the Twiss and dispersion parameters $\beta_{0}, \alpha_{0}, \eta_{0}$, and $\eta_{0}^{\prime}$ at the entrance of the outer bend, and the strength $k_{q f a}$ of the quadrupole QFA between outer and inner bends, $\langle\mathcal{H}\rangle_{o}$ and $\langle\mathcal{H}\rangle_{i}$ can be easily evaluated using Eq. (8). Thus, the horizontal emittance $\epsilon_{x}$ is the function of 5 parameters $\beta_{0}, \alpha_{0}, \eta_{0}, \eta_{0}^{\prime}$, and $k_{q f a}$.

Because of the constraint of the mirror symmetric structure, the Twiss parameter $\alpha_{i c}$ and the dispersion function $\eta_{i c}^{\prime}$ should be equal to zero at the center of the inner bend, i.e., $\alpha_{i c}=0$ and $\eta_{i c}^{\prime}=0$. Applying the constraint $\eta_{i c}^{\prime}=0$, we can solve the quadrupole QFA strength $k_{q f a}$ as functions of $\eta_{0}$ and $\eta_{0}^{\prime}$. Applying the constraint $\alpha_{i c}=0$, we can also solve $\alpha_{0}$ as a function of $\beta_{0}$. However, to avoid solving $\alpha_{0}$, we use the beta function $\beta_{i c}$ at the center of the inner bend as the free variable instead of $\beta_{0}$, and express $\beta_{0}$ and $\alpha_{0}$ as functions of $\beta_{i c}, \eta_{0}$, and $\eta_{0}^{\prime}$. Thus, the five parameter emittance $\epsilon_{x}\left(\beta_{0}, \alpha_{0}, \eta_{0}, \eta_{0}^{\prime}, k_{q f a}\right)$ function reduces to the three parameter function $\epsilon_{x}\left(\beta_{i c}, \eta_{0}, \eta_{0}^{\prime}\right)$.

The emittance $\epsilon_{x}$ vs the beta function $\beta_{0}$ for given dispersion functions $\eta_{0}$ and $\eta_{0}^{\prime}$ at the entrance of outer bends is shown in Fig. 3. For each pair of dispersion

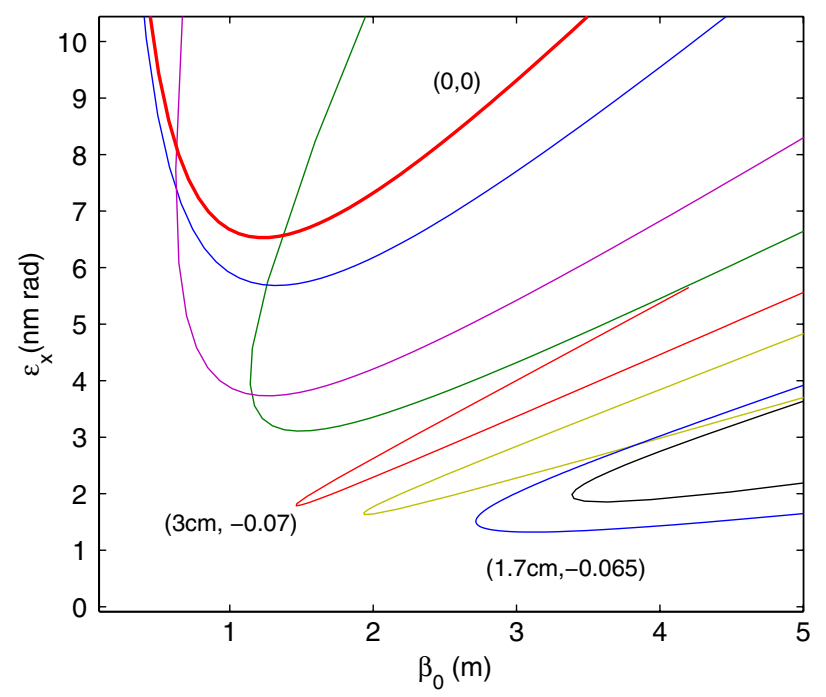

FIG. 3. Horizontal natural emittance $\epsilon_{x}$ of the ALS storage ring as a function of $\beta_{0}$ for given dispersion functions $\eta_{0}$ and $\eta_{0}^{\prime}$. The different curves represent different pairs $\left(\eta_{0}, \eta_{0}^{\prime}\right)$. 


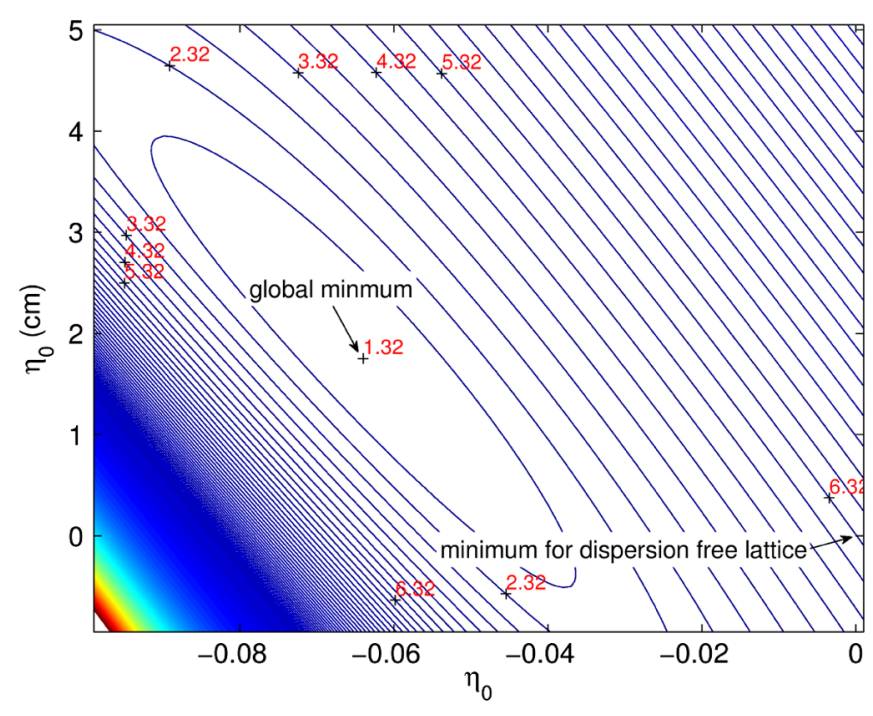

FIG. 4. Local minimum horizontal emittance $\epsilon_{x}$ as functions of dispersion functions $\eta_{0}$ and $\eta_{0}^{\prime}$. The labels for contour lines represent their respective emittance in $\mathrm{nm}$ rad. The locations of the minimum emittance for the dispersion free lattice and the global minimum emittance are indicated in the plot.

functions $\left(\eta_{0}, \eta_{0}^{\prime}\right)$, we could find a local minimum emittance. For dispersion free ALS lattice, i.e., $\left(\eta_{0}, \eta_{0}^{\prime}\right)=$ $(0,0)$, the theoretical minimum emittance we can achieve is about $6 \mathrm{~nm}$ rad. Relaxing the constraint of the dispersion function, the emittance can be reduced. The local minimum emittance as functions of dispersion function pair $\left(\eta_{0}, \eta_{0}^{\prime}\right)$ is shown in Fig. 4 . We can see the global minimum emittance we can achieve is about $1.3 \mathrm{~nm}$ rad when $\left(\eta_{0}, \eta_{0}^{\prime}\right)=(1.7 \mathrm{~cm},-0.065)$.

Having the desired values of the ring emittance and its associated Twiss parameters and dispersion functions at the entrance of the outer dipole, the strength of quadrupoles QF and QD in the straight are determined using a lattice matching technique with the constraints $\alpha=0$ and $\eta^{\prime}=0$ at the center of the straight. After obtaining all the quadrupole strengths, the stability of the ring is checked, and Twiss and dispersion functions at the center of the straight are calculated. The horizontal emittances vs beta functions at the center of the straight are shown in Fig. 5. For comparison, the solutions optimized using a genetic algorithm are also shown in the plot. A good agreement between them is observed. The TME method gives all the possible solutions, while the genetic algorithms only give the optimal ones. It is obvious that, for a given emittance, there are two distinct solution regions: one has a large beta function giving the lattice used for the baseline upgrade [Fig. 1(b)], and the other has a low-beta function which can be used for ALS ultimate upgrade [Fig. 1(c)].

It is worth pointing out that from Fig. 4 we can see that the theoretical minimum emittance we can achieve for the ALS lattice is about $1.3 \mathrm{~nm}$ rad. However, from Fig. 5, we see that the minimum emittance is about $1.7 \mathrm{~nm} \mathrm{rad}$. This is

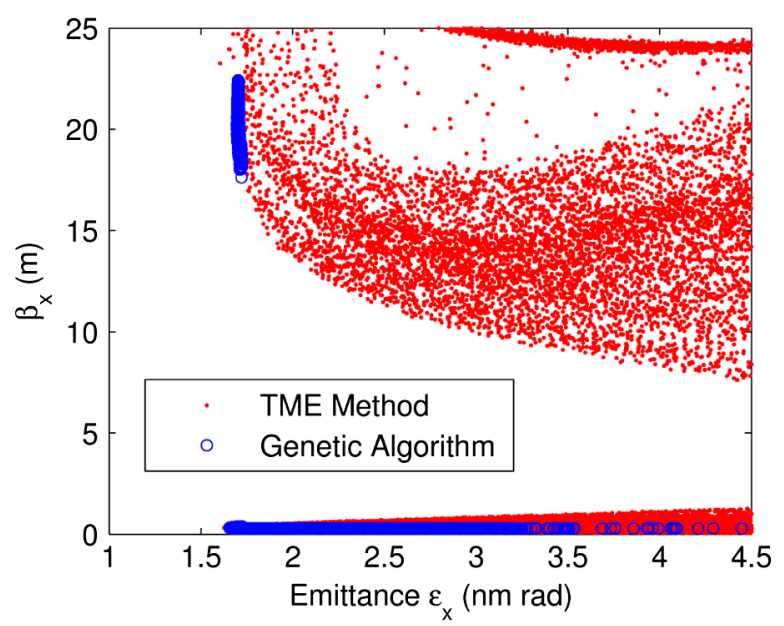

FIG. 5. Lattice solutions optimized using two different techniques, the TME method represented by dots and the genetic algorithms represented by circles.

because some solutions shown in Fig. 4 do not exist when determining the quadrupole strengths of QF and QD using the lattice matching technique. If additional quadrupole families are added to the straight we may restore the lost solutions.

\section{DYNAMIC APERTURE OPTIMIZATION}

Optimization of dynamic aperture is a challenging aspect of storage-ring lattice design. A large dynamic aperture is required for efficient injection and long beam lifetime. Several methods have been successfully applied by lattice designers to optimize the dynamic aperture of the storage ring. The resonance driving term minimization is one of these methods. In this method the nonlinear Hamiltonian of particle motion is written as a series of driving terms of different orders in field gradients [21]. In general, each sextupole and quadrupole contributes to a driving term as a complex number in the overall summations. By properly choosing the sextupole strength, these driving terms can be minimized, thus nonlinear effects are reduced. To successfully use this method, the designer must carefully choose weight factors for many resonance driving terms, based upon experiences or even by guessing.

Another widely used method is the brute force sextupole scan technique [19]. This technique scans all the sextupole settings, and keeps the setting with the largest dynamic aperture. Although this method is straightforward and easily implemented, it is only good for a ring with a small number of sextupoles since the computing time of this method exponentially depends on the sextupole number.

The third method is genetic optimization, which can address the limitation of the sextupole scan method [19]. In this section, we will apply this technique to optimize the dynamic aperture of ALS ultimate upgrade lattice. Instead of the commonly used dynamic aperture area, we have 
used the total diffusion rate as an objective in the optimization.

\section{A. Objectives}

To optimize the dynamic aperture of a storage-ring lattice using either brute force scan or genetic algorithms (GA), first we need to properly choose quality factors, i.e., the optimization objectives. The most commonly used objective by ring designers is the dynamic aperture area. The evaluation of this objective relies heavily upon the numerical particle tracking technique. Particles with different initial coordinates in $x-y$ configuration space are tracked for multiple turns. If the particles survive, the initial coordinates of the particles are recorded. In the end, we could have the initial coordinates of all stable particles. The area which encompasses these initial coordinates, known as the dynamic aperture area, is often used as a quality factor to compare the nonlinear performance of the lattice. It is generally believed that the larger the aperture area the better the dynamic properties the lattice has. However, the dynamic aperture area could not reveal the detailed nonlinear behavior of particles, and the resonance structures of ring lattice.

Frequency map analysis (FMA) [22] can address these limitations. This technique has been introduced to study dynamics of particle accelerator for more than 15 years. Briefly speaking, FMA constructs a map between $x-y$ configuration space and $\nu_{x}-\nu_{y}$ tune space by tracking particles with different initial coordinates for a period time. For each test particle, the discrete trajectories are recorded at an observation location. Using the numerical analysis of fundamental frequencies algorithm [23], we can precisely calculate the tunes of this particle. As a stability index, the diffusion rate of particle trajectory is defined as follows:

$$
d=\log \left(\sqrt{\frac{\left(\nu_{x, 1}-\nu_{x, 2}\right)^{2}+\left(\nu_{y, 1}-\nu_{y, 2}\right)^{2}}{N}}\right),
$$

where $\nu_{x, 1}$ and $\nu_{y, 1}$ represent the horizontal and vertical tunes calculated for the first $N$ turns tracking data; $\nu_{x, 2}$ and $\nu_{y, 2}$ represent the horizontal and vertical tunes calculated for the following $N$ turns. Because of the logarithmic scale, the diffusion rate defined in Eq. (11) is always negative. A large negative number indicates that the diffusion is small and the particle trajectory is stable, while a small negative number indicates the diffusion is large and the particle motion is irregular.

Incorporating the diffusion rate calculation to the dynamic aperture evaluation, we can obtain a powerful tool to study the nonlinear dynamic performance of a storage ring. For each test particle in $x-y$ configuration space, not only its survival status is recorded, but also its diffusion rate is calculated. The total diffusion rate is then given by the summation of all the particle diffusion rates. The idea of using total diffusion rate as an objective to optimize dynamic aperture of a lattice is not new [6,24-26]. However, we successfully implemented it in the genetic optimization. Instead of maximizing dynamic aperture area, if it is possible to minimize the total diffusion rate, the optimized lattice will have excellent dynamic performance. Next, we are going to illustrate the dynamic aperture optimization using genetic algorithms (GA) with total diffusion rate as an objective.

\section{B. Optimization}

There are many different ways to implement genetic algorithms [27]. The nondominated sorting genetic algorithm II (NSGA-II) is considered as the most effective one. The detailed discussion of this algorithm is out of the scope of this paper, and can be found in paper [28]. Here, we only focus on their application to optimize the dynamic aperture of storage-ring lattices. The lattice we are going to optimize is the one shown in Fig. 1(c). The variables used for the optimization are sextupole strengths. At the current stage, the ALS storage-ring lattice has two chromatic sextupole families ("SF" and "SD") in arcs. However, when the baseline upgrade is finished, an additional four harmonic sextupoles ("SHF," "SHD," "SHF1," and "SHD1") will be installed in straight sections of each sector [5]. The arrangement of these chromatic and harmonic sextupole is shown in Fig. 1. The strengths of chromatic sextupoles are determined by the chromaticity fitting. Thus, in this optimization problem, there are four free parameters, i.e., the strengths of the harmonic sextupoles.

To compare the optimization performance, both the dynamic aperture area and total diffusion rate are used as optimization objectives. An accelerator modeling code, such as GOEMON[29] and ELEGANT[30], can be used for particle tracking and objective evaluations.

Figure 6 shows solutions at different generations in the objective spaces for the optimizations using (a) dynamic aperture area as objective and (b) total diffusion rate as objective. In either case, the objectives are calculated for both on- and off-momentum $(d p / p=0.5 \%)$ particles. The particles are tracked through the lattice with quadrupole strength and roll errors. The magnitudes of these errors are $0.03 \%$ and $0.5 \mathrm{mrad}$, respectively. For the dynamic aperture area calculations, the 21-line search method [30] is used and particle is tracked for 512 turns; the boundary of the aperture is clipped to avoid island before calculating the aperture area. For the total diffusion rate calculation, the particles are launched over nonuniform 21 by 21 grids in $x-y$ space and tracked for 512 turns (the nonuniform grids in $x-y$ space lead to an equal spacing in action space). For the surviving particles, the diffusion rates are calculated according to Eq. (11). If the particles are lost, the diffusion rates are assigned to a value which is slightly larger than the largest diffusion rate for surviving particles. In this 


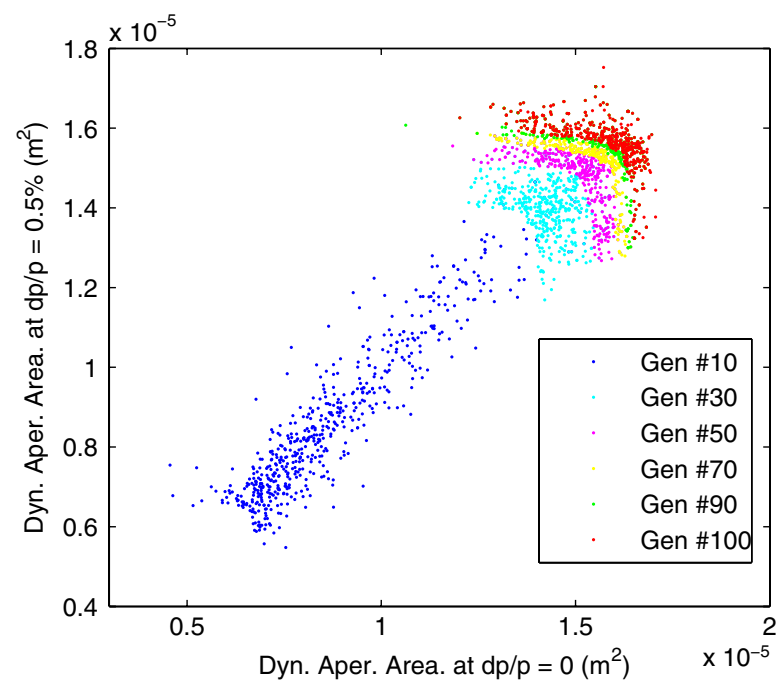

(a)

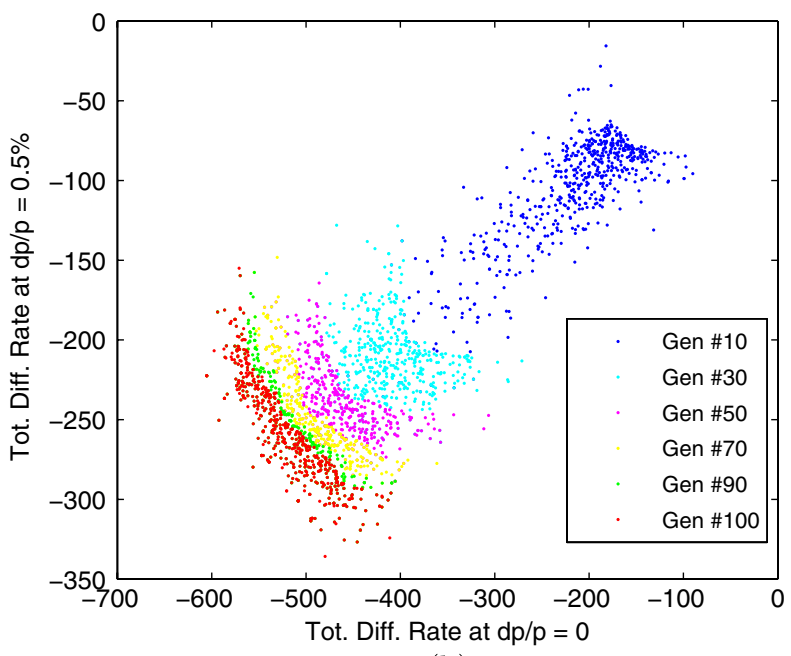

(b)

FIG. 6. Lattice solutions at different generations in objective space: (a) optimization using dynamic aperture area as objective; (b) optimization using total diffusion rate as objective.

problem, the number -3 is used. Then, the total diffusion rate is then given by the summation of all the diffusion rates over the 21 by 21 grids. Figure 6 shows that the solutions converge after 100 generations, and optimal solution fronts are obtained for either case.

The dynamic apertures and diffusion rates of example lattices from the solution fronts are shown in Fig. 7 for (a) optimization using aperture area as objective and (b) optimization using total diffusion rate as objective. The diffusion rates of particle motions are represented by color in the figure. The blue color indicates that the particle orbit is regular, while the red color indicates irregular. We can see that these two optimal lattices have almost the same aperture area. However, the lattice optimized using total diffusion rate as objectives has a superior dynamic performance, because in Fig. 7(b) there is less red color and some resonance structures disappear.

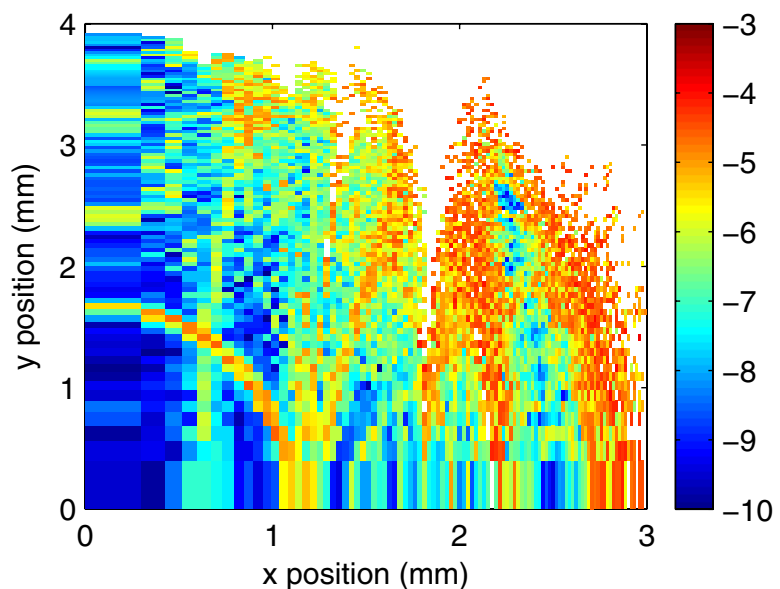

(a)

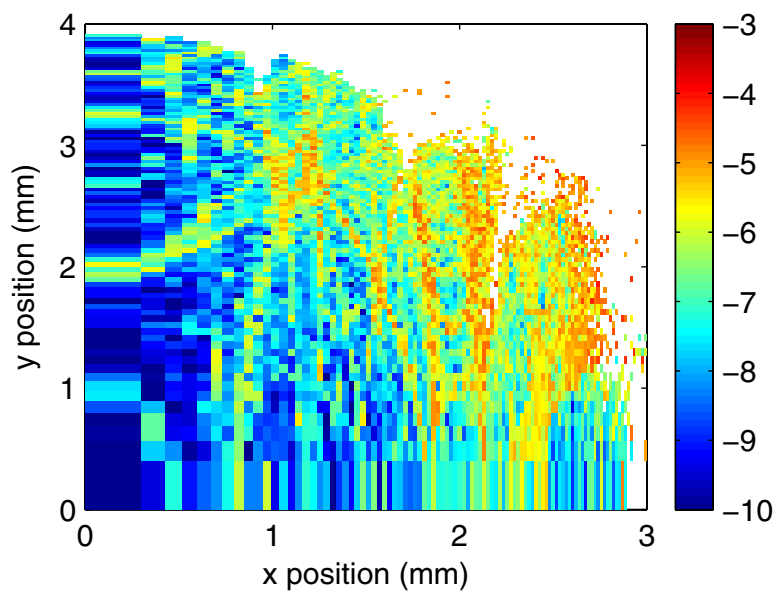

(b)

FIG. 7. Dynamic apertures and diffusion rates of lattice optimized using (a) dynamic aperture area as objective or (b) total diffusion rate as objectives. The diffusion rate of particle motions is color coded in the plot. The blue color represents the particle is very stable, and the red color represents very chaotic.

\section{SIMULTANEOUS LINEAR AND NONLINEAR LATTICE OPTIMIZATION}

From Secs. III and IV, we can see that the lattice optimization proceeds in two stages. The first stage is to design a linear lattice to meet some property requirements, such as small-emittance and low-beta functions, by changing the quadrupole settings. The second stage is to optimize the nonlinear properties of this lattice by changing the sextupole settings. These two stages are well separated. However, without a consideration of the suppression of nonlinear effects at the first stage, the subsequent optimization in the second stage might not succeed. In this case, the linear solution found in the first stage needs to be revisited by changing the working tunes to ensure the best overall optimization. Such a strategy, known as DATS, has been widely used to find a best working point in many facilities [31,32]. There are also many successful examples of using the first stage to mitigate 
strong nonlinear effects by canceling geometric driving terms, which are achieved by properly setting the phase advance over sections of lattice [33-36].

In this section, we explore optimizing the linear and nonlinear properties of lattice simultaneously using NSGA-II. The lattice we are going to optimize is the same as the one in Sec. IV. It has 12 superperiods with three quadrupole (QF, QD, and QFA), two chromatic sextupole (SF and SD), and four harmonic sextupole (SHF, SHD, SHF1, and SHD1) families. The three quadrupole strengths are used as parameters for the linear property optimizations, and four harmonic sextupoles are used for nonlinear property optimizations, and two chromatic sextupoles are used for chromaticity fittings. The objectives we want to optimize are two linear properties (horizontal beta function $\beta_{x}$ at the center of straight and horizontal natural emittance $\epsilon_{x}$ ), and one nonlinear property (the

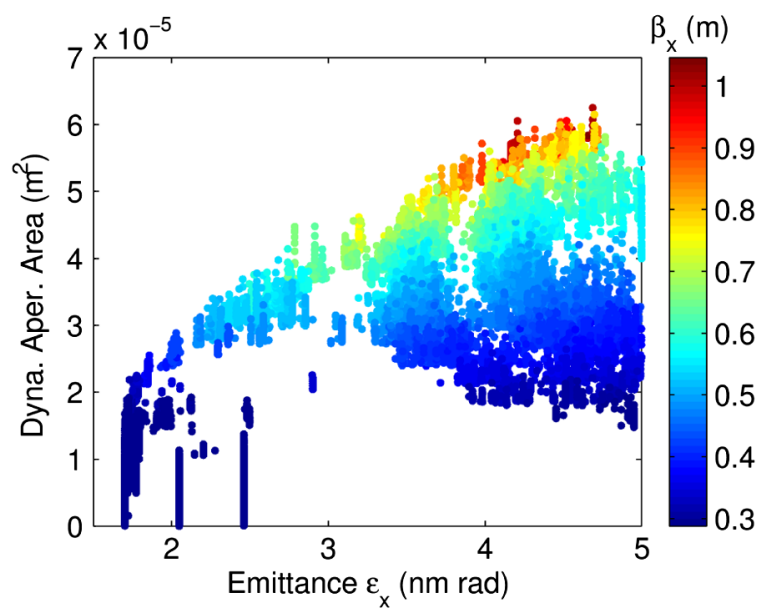

(a)

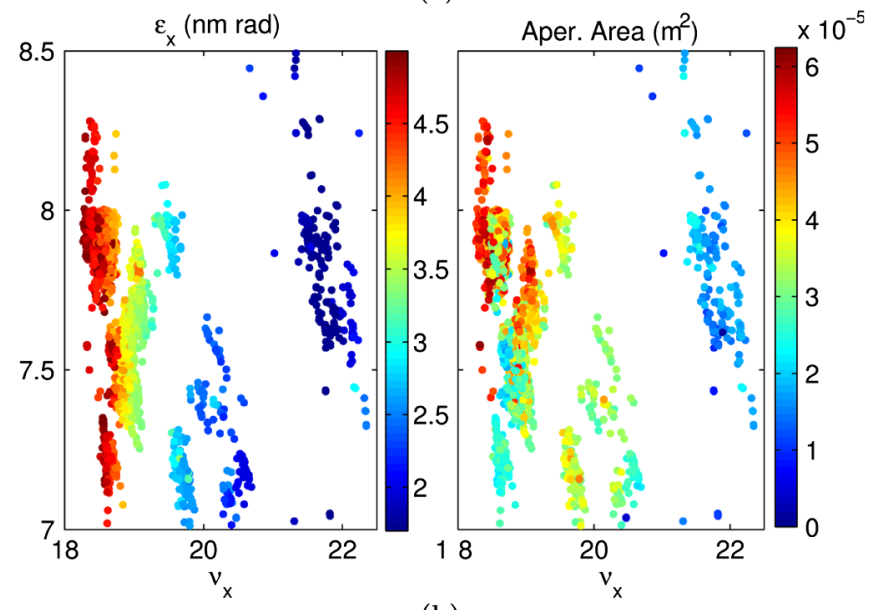

(b)

FIG. 8. Linear and nonlinear optimizations of ALS lattice using NSGA-II (a) solution front in the objective spaces, i.e., the horizontal emittance $\epsilon_{x}$ ( $x$ axis), dynamic aperture area ( $y$ axis), and horizontal beta function $\beta_{x}$ (color coded); (b) solutions in the tune space $\nu_{x}$ and $\nu_{y}$, the horizontal emittance (left) and dynamic aperture area (right) are colored. dynamic aperture area). The constraints are to ensure the stability of the lattice, positive damping, and reasonable maximum Twiss and dispersion functions, and vertical phase advance.

The optimal solutions in the objective spaces are shown in Fig. 8(a). For this optimization problem, 20000 populations and 400 generations are used, and it takes about 70 hours with $64 \mathrm{CPUs}$ to reach these solution fronts. In the figure, we can clearly see that there are trade-offs between small-emittance $\left(\boldsymbol{\epsilon}_{x}\right)$, large dynamic aperture and small horizontal beta function $\left(\beta_{x}\right)$. In the previous section, the lattice we try to optimize has the horizontal emittance of about $1.6 \mathrm{~nm}$ rad. Figure 8 (a) shows that the dynamic aperture we can achieve for this lattice is about $2 \times$ $10^{-5} \mathrm{~m}^{2}$. If we allow emittance increase to $2.5 \mathrm{~nm} \mathrm{rad}$, the dynamic aperture is almost doubled. Figure 8(b) shows the solutions in the tune space. It can be seen that, for the lattices with the emittance less than $2 \mathrm{~nm} \mathrm{rad}$, the work tunes $\nu_{x}$ and $\nu_{y}$ are between 21-22 and 7.5-8, and the dynamic aperture areas are about $2 \times 10^{-5} \mathrm{~m}^{2}$, while for the lattice with the emittance of about $2.5 \mathrm{~nm} \mathrm{rad}$, the working tunes are between 20-21 and 7-7.5, and the dynamic aperture areas are about $3.5 \times 10^{-5} \mathrm{~m}^{2}$. These optimal solution fronts provide us guidance to choose a candidate lattice for ALS future upgrade.

\section{CONCLUSIONS}

We have successfully developed systematic strategies and techniques to design and optimize the ALS storagering lattice for its potential future upgrades. First, the TME theory is applied to optimize small-emittance and low-beta lattices. The study provides us a different perspective on the lattice design, and confirms results found in earlier study using both global scan of all stable settings (GLASS) and MOGA techniques. Then, the dynamic aperture of the lattice is optimized using MOGA with total diffusion rate as an optimization objective. It is illustrated that this optimization can lead to a superior nonlinear dynamic performance. The linear and nonlinear properties of the lattice are also optimized simultaneously, and tradeoffs are found among the low emittance, the small beta function and the large dynamic aperture. These trade-offs can provide us a guideline to choose a candidate lattice for ALS future upgrades.

The ultimate ALS small emittance and low-beta lattice presented in this paper may have a dynamic aperture too small to allow traditional off-axis injection. To address this problem, a novel pulsed multipole injection scheme has been proposed at the ALS [37]. Alternatively, an alternating high and low beta lattice, which has a large $\beta_{x}$ function at the injection straight, could be used for ALS future upgrades. So far, we have successfully applied MOGA to design and optimize this lattice. The feasibility of using the above two approaches to lower the ALS emittance is still under investigation. 


\section{ACKNOWLEDGMENTS}

The authors would like to thank F. Sannibale and C. Papadopoulos at Lawrence Berkeley National Lab, and L. Yang at Brookhaven National Lab for fruitful discussions. This work is supported by the Director Office of Science of the U.S. Department of Energy under Contract No. DEAC02-05CH11231.

[1] A. Jackson, in Proceedings of the Particle Accelerator Conference, Washington, DC, 1993 (IEEE, Washington, 1993), pp. 1432-1435.

[2] D. Robin et al., in Proceedings of the Particle Accelerator Conference, Chicago, IL, 2001 (IEEE, New York, 2001), pp. 2632-2634.

[3] C. Steier et al., in Proceedings of the 23rd Particle Accelerator Conference, Vancouver, Canada, 2009 (IEEE, Piscataway, NJ, 2009), TU5RFP042.

[4] H. Nishimura, S. Marks, D.S. Robin, R. D. Schlueter, C. A. Steier, and W. Wan, in Proceedings of the 16th IEEE International Pulsed Power Conference, Albuquerque, NM (IEEE, Piscataway, NJ, 2007), pp. 1170-1172.

[5] C. Steier et al., in Proceedings of the IPAC'10 Conference, Kyoto, Japan (ICR, Kyoto, 2010), pp. 2645-2647.

[6] C. Steier et al., Nucl. Instrum. Methods Phys. Res., Sect. A 649, 25 (2011).

[7] L. C. Teng, Fermilab Report No. TM-1269, 1984.

[8] S. Y. Lee, Phys. Rev. E 54, 1940 (1996).

[9] D. S. Robin, W. Wan, F. Sannibale, and V. P. Suller, Phys. Rev. ST Accel. Beams 11, 024002 (2008).

[10] L. Yang, D. Robin, F. Sannibale, C. Steier, and W. Wan, Nucl. Instrum. Methods Phys. Res., Sect. A 609, 50 (2009).

[11] H. Tanaka and A. Ando, Nucl. Instrum. Methods Phys. Res., Sect. A 369, 312 (1996).

[12] Y. Papaphilippou and P. Elleaume, in Proceedings of the 21st Particle Accelerator Conference, Knoxville, 2005 (IEEE, Piscataway, NJ, 2005), pp. 2086-2088.

[13] C.X. Wang, Phys. Rev. ST Accel. Beams 12, 061001 (2009).

[14] M. Borland, L. Emery, H. Shang, and R. Soliday, in Proceedings of the 20th Particle Accelerator Conference, Portland, OR, 2003 (IEEE, New York, 2003), pp. 3461-363.

[15] T. Tanaka and H. Kitamura, J. Synchrotron Radiat. 8, 1221 (2001).

[16] S. Ramberger and S. Russenschuck, in Proceedings of the 6th European Particle Accelerator Conference, Stockholm, 1998 (IOP, London, 1998), pp. 2014-2016.

[17] D. Schirmer, M. V. Hartrott, S. Khan, D. Krmer, and E. Weihreter, in Proceedings of the Particle Accelerator
Conference, Dallas, TX, 1995 (IEEE, New York, 1995), pp. 1879-1881.

[18] I. V. Bazarov and C. K. Sinclair, Phys. Rev. ST Accel. Beams 8, 034202 (2005).

[19] M. Borland, V. Sajaev, L. Emery, and A. Xiao, in Proceedings of the 23rd Particle Accelerator Conference, Vancouver, Canada, 2009 (Ref. [3]), TH6PFP062.

[20] R. H. Helm, M. J. Lee, and P. L. Morton, in Proceedings of the 5th IEEE Particle Accelerator Conference, San Francisco, CA, 1973 [IEEE Trans. Nucl. Sci. NS-20, No. 3 (1973)], p. 900.

[21] J. Bengtsson, SLS Note No. 9/97, 1997.

[22] H. S. Dumas and J. Laskar, Phys. Rev. Lett. 70, 2975 (1993).

[23] J. Laskar, Icarus 88, 266 (1990).

[24] C. Steier and W. Wan, in Proceedings of the IPAC'10 Conference, Kyoto, Japan (Ref. [5]), pp. 4746-4748.

[25] Y. Papaphilippou and F. Zimmermann, Phys. Rev. ST Accel. Beams 2, 104001 (1999).

[26] Y. Papaphilippou, in Proceedings of the Particle Accelerator Conference, Chicago, IL, 2001 (Ref. [2]), pp. 462-464.

[27] A. Konak, D. W. Coit, and A.E. Smith, Reliability Engineering and System Safety 91, 992 (2006) [http:// dx.doi.org/10.1016/j.ress.2005.11.018].

[28] K. Deb, IEEE Trans. Evolutionary Comput. 6, 182 (2002) [http://ieeexplore.ieee.org/xpls/abs_all.jsp?arnumber=996017].

[29] H. Nishimura, in Proceedings of the Particle Accelerator Conference, Chicago, IL, 2001 (Ref. [2]), pp. 3066-3068.

[30] M. Borland, Advanced Photon Source Report No. LS-287, 2000.

[31] D. Einfeld, in Proceedings of the 11th European Particle Accelerator Conference, Genoa, 2008 (EPS-AG, Genoa, Italy, 2008), pp. 3140-3142.

[32] L. Jin and F. Schmidt, Report No. LHC-PROJECT-NOTE182.

[33] A. Verdier, in Proceedings of the 18th Particle Accelerator Conference, New York, 1999 (IEEE, New York, 1999), p. 398.

[34] S. L. Smith, in Proceedings of the 8th European Particle Accelerator Conference, Paris, 2002 (EPS-IGA and CERN, Geneva, 2002), pp. 44-48.

[35] R. Hettel et al., in Proceedings of the 18th Particle Accelerator Conference, New York, 1999 (Ref. [33]), pp. 206-208.

[36] A. Wolski, in Proceedings of the European Particle Accelerator Conference, Vienna, 2000 (EPS, Geneva, 2000), pp. 1095-1097.

[37] C. Sun, Z. Fisher, C. Pappas, and D. Robin, in Proceedings of the IPAC'10 Conference, Kyoto, Japan (Ref. [5]), WEPEA068. 